Copyright by the Cambridge University Press
A. DOUGALL, C. SHILTON, J. LOW CHOY, B. ALEXANDER and S. WALTON (2009). New reports of Australian cutaneous leishmaniasis in Northern Australian macropods. Epidemiology and Infection, 137, pp 1516-1520. doi:10.1017/S0950268809002313. 


\title{
SHORT REPORT \\ New reports of Australian cutaneous leishmaniasis in Northern Australian macropods
}

\author{
A. DOUGALL ${ }^{1,2 *}$, C. SHILTON ${ }^{3}$, J. LOW CHOY ${ }^{4}$, B. ALEXANDER ${ }^{5}$ \\ AND S. WALTON ${ }^{1,2}$ \\ ${ }^{1}$ Menzies School of Health Research, Charles Darwin University, Darwin, Northern Territory, Australia \\ ${ }^{2}$ Institute of Advanced Studies, Charles Darwin University, Darwin, Northern Territory, Australia \\ ${ }^{3}$ Berrimah Veterinary Laboratories, Department of Primary Industry, Fisheries and Mines, Darwin, \\ Northern Territory, Australia \\ ${ }^{4}$ Territory Wildlife Park, Berry Springs, Northern Territory, Australia \\ ${ }^{5}$ Liverpool School of Tropical Medicine, Liverpool, UK
}

(Accepted 30 January 2009; first published online 17 March 2009)

\section{SUMMARY}

Cutaneous leishmaniasis caused by various species of Leishmania is a significant zoonotic disease in many parts of the world. We describe the first cases of Australian cutaneous leishmaniasis in eight northern wallaroos, one black wallaroo and two agile wallabies from the Northern Territory of Australia. Diagnosis was made through a combination of gross appearance of lesions, cytology, histology, direct culture, serology and a species-specific real-time PCR. The causative organism was found to be the same unique species of Leishmania previously identified in red kangaroos. These clinical findings provide further evidence for the continuous transmission of the Australian Leishmania species and its presence highlights the importance of continued monitoring and research into the life-cycle of this parasite.

Key words: Cutaneous leishmaniasis, diagnosis, macropods, Northern Territory.

The Leishmania spp. are protozoan parasites which naturally infect many different species of mammal worldwide. They are the causative agent for zoonotic disease and the most common form, cutaneous leishmaniasis (CL), is believed to affect some $1-1.5$ million people every year, with a further 350 million at risk of infection. A potentially fatal form known as visceral leishmaniasis (VL) affects another 500000 people anually. Those affected are predominantly from poor or marginalized countries and it is widely believed that disease prevalence is grossly underestimated. The Leishmania parasite has a dimorphic life-cycle, being able to exist either intracellularly in the macrophages

* Author for correspondence: Miss A. Dougall, Menzies School of Health Research, PO Box 41096, Casuarina, NT 0811, Australia. (Email: Annette.Dougall@cdu.edu.au) of its mammalian host or extracellularly within the gut of a phlebotomine sand-fly vector [1].

The first report of CL in Australia was published in 2004 [2]. Until this report Australia and Antarctica were the only continents considered free of Leishmania parasites. The Australian cases of CL were diagnosed in captive red kangaroos (Macropus rufus) and restricted to the rural Darwin area of the Northern Territory (NT). Red kangaroos are widespread across much of inland Australia but they are not native to the more humid northern tropical regions and the disease was seen as an opportunistic infection. The causative species was characterized as a unique species of Leishmania likely to be endemic to Australia. We now report the first Australian clinical cases of CL in three other captive species, i.e. northern wallaroo 


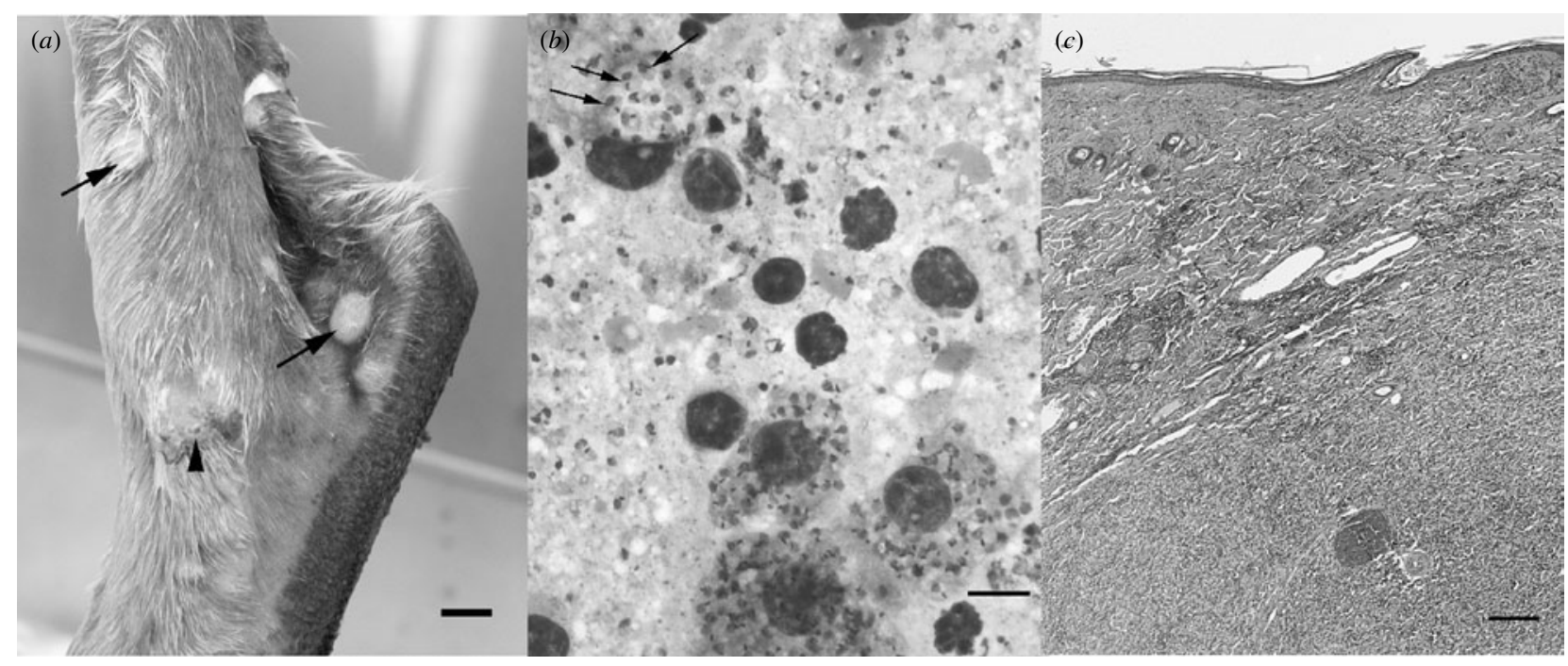

Fig. 1. (a) Medial hock of a northern wallaroo depicting multiple smooth nodules (arrows) and one ulcerated and encrusted lesion (arrowhead) (scale bar, $1 \mathrm{~cm}$ ). (b) Impression smear from a northern wallaroo skin lesion depicting four infected macrophages among lymphocytes and cellular debris. Arrows point to three individual Leishmania amastigotes, each with round dark nucleus and single kidney kinetoplast. Diff-quik stain (Lab Aids Pty Ltd) (scale bar, $10 \mu \mathrm{m}$ ). (c) Histological section of skin lesion from a northern wallaroo depicting marked deep dermal infiltration with macrophages resulting in expansion of the dermis and obliteration of normal dermal structures in the lower right portion of the panel. The infiltrate wanes towards the left upper region of the panel, where the dermis is moderately infiltrated with primarily lymphocytes and plasma cells. Haematoxylin and eosin stain (scale bar, $200 \mu \mathrm{m}$ ).

(Macropus robustus woodwardi), black wallaroo (M. bernardus) and agile wallaby (M. agilis agilis).

Skin lesions suggestive of CL were observed in eight captive northern wallaroos housed together in a monsoonal open woodland habitat at a facility in the rural Darwin area in the NT of Australia. The wallaroos had been in captivity for 9 years before showing clinical signs of $C L$ which have been ongoing since. Northern wallaroos are macropods native to the tropical regions of the NT and northeastern Western Australia. Their natural habitat is typically associated with drier rocky escarpment and hilly country [3]. The lesions were located distally in areas concentrated on the tail, inner forearms, hind legs and ears. Grossly, lesions on the limbs and tail appeared as focal to coalescing $0 \cdot 5-2 \mathrm{~cm}$ diameter areas of thickened skin or raised variably encrusted or ulcerative pale nodules (Fig. 1). On ears, lesions involved primarily the distal margins of the pinnae which were irregular in outline, were mildly swollen and variably encrusted. The severity and number of lesions varied in the affected individuals. Interestingly, lesions in two animals were observed on the cloaca, evidence of possible mucosal infection. For the index case, amastigotes were seen by microscopic examination of Diff-Quik (Lab Aids
Pty Ltd, Australia)-stained impression smears of lesions (Fig. 1). Histological examination of the affected skin from the index northern wallaroo case was prepared in standard fashion and $5-\mu \mathrm{m}$-thick sections were stained with haematoxylin and eosin. Lesions were characterized by moderate to marked infiltration of the superficial and/or deep dermis with macrophages containing abundant amastigotes. There were moderate numbers of plasma cells and lymphocytes intermingled with macrophages present in the dermis and at the margins of lesions (Fig. 1).

The diagnosis in this case and a further three cases was confirmed by direct culture from the tissue of various lesions or swollen ear margins using standard Leishmania culture in NNN medium [2]. DNA extraction was carried out from various skin lesions, spleen, lymph nodes or cloaca using a commercially available kit (DNeasy Tissue kit, Qiagen Ltd, Australia) according to the manufacturer's instructions. These tissue samples were analysed by a Taqman probe species-specific real-time PCR targeting the mini-exon gene from the kangaroo Leishmania [2]. Reactions were carried out using $1 \times$ Invitrogen ${ }^{\mathrm{TM}}$ Platinum ${ }^{\mathbb{R}}$ Quantitative PCR SuperMix-UDG (Invitrogen, Australia) using $0.3 \mu \mathrm{M}$ of 
primers rooME-F2 5'-AAACTTCCGGAACCTGTCGT-3' and rooME-R2 5'-GTAGGCACCCGAAGAGACC-3' and $0.05 \mu \mathrm{m}$ Taqman probe $5^{\prime}$-d FAM-CCGGCAAGATTTTGGGAGCG-BHQ-1-3'

(Biosearch Technologies Inc., USA). $\mathrm{MgCl}_{2}$ was used at the final concentration of $6 \mathrm{~mm}$ with the addition of $1 \times$ Q solution (Qiagen Ltd). Cycling and fluorescent detection was $2 \mathrm{~min}$ at $50^{\circ} \mathrm{C}, 2 \mathrm{~min}$ at $95^{\circ} \mathrm{C}$ followed by 35 cycles: $15 \mathrm{~s}$ at $95^{\circ} \mathrm{C}$ and $40 \mathrm{~s}$ at $60{ }^{\circ} \mathrm{C}$ acquiring to cycling A Green using the Rotor gene 2000 or 6000 (Corbett Life Science, Australia). All skin lesion tissue sampled from six cases including the four cases from which the organism was cultured, were positive using this real-time PCR. Lymph node was positive from $2 / 2$ samples and spleen tissue was positive in $1 / 2$ samples. One animal with lesions seen on the cloaca was positive for real-time PCR by NNN medium culture using tissue taken directly from this region confirming a mucosal infection.

Sera collected from $7 / 8$ cases were also positive using an enzyme-linked immunosorbent assay (ELISA). This assay demonstrated specific IgG antibodies to soluble Leishmania antigen (SLA) prepared using cultured promastigotes from northern wallaroo lesions. SLA was prepared by washing cultures three times with $1 \times \mathrm{PBS} ; 1 \times 10^{8} / \mathrm{ml}$ of promastigotes were then freeze-thawed five times. For ELISA the soluble antigen was applied for $2 \mathrm{~h}$ at $37^{\circ} \mathrm{C}$ using a $1 / 100$ dilution in carbonate buffer ( $\mathrm{pH} 9 \cdot 6$ ) to a 96-well plate. The SLA was blocked with $3 \% \mathrm{BSA} / 0 \cdot 05 \%$ Tween-20 and serum was applied at a 1/600 dilution. Bound serum IgG was then detected using a $1 / 2000$ dilution of pProtein A conjugated to horseradish peroxidise (Sigma-Aldrich Pty Ltd, Australia). Binding was detected using 3,3',5,5'-tetramethylbenzidine substrate for ELISA (Sigma-Aldrich Pty Ltd). Opportunistic tissue or blood collection was not possible for one case although the symptoms were consistent with those seen in the other animals.

Molecular diversity was investigated in two cases using PCR amplification and fluorescent sequencing with published primers for the Leishmania miniexon gene [4]. Results confirmed sequence match with the original Leishmania sp. from red kangaroos [2].

In addition we report for the first time Australian $\mathrm{CL}$ in one black wallaroo and two agile wallabies housed in separate enclosures at the same facility. These enclosures were within $2 \mathrm{~km}$ of those housing the northern wallaroos with $\mathrm{CL}$ as well as the previously documented red kangaroos [2]. In the black wallaroo and agile wallabies symptoms of CL were seen about 6 months after an initial investigation for disease in the northern wallaroos. As with the northern wallaroos, the black wallaroo had been in captivity for some years before showing clinical signs of CL. In the wild its range is restricted to steep rocky escarpment and plateau country of west and central Arnhem Land in the NT. The captive environment is open monsoonal woodland with agile wallabies naturally abundant in the area. The black wallaroo exhibited lesions on the ears similar to the appearance in the northern wallaroos. It also had small nodular lesions around the eyes, again indicating possible mucosal involvement of the parasite. The IgG response was positive to SLA and ear tissue was positive by species-specific real-time PCR. The two agile wallabies showed less severe symptoms with lesions appearing predominantly on the ears. Ear tissue for both animals was positive using species-specific real-time PCR and they showed high serological responses to SLA. Agile wallabies are the most common macropod species in the Darwin rural area and are abundant in the bush surroundings of the captive facility. They have a gregarious nature and are easily maintained in captivity and on public display. The two agile wallaby cases were juveniles recently introduced to the captive environment, thus it may have been their first exposure to Leishmania. Lesions have subsequently healed for the agile wallabies involved in this study. This suggests an inherent immunity in these animals. Further epidemiological studies are needed to establish their possible role as reservoirs of Australian Leishmania.

Naivety to infection and subclinical stress related to an incompatibility with their captive conditions may have contributed to the varying severity of disease seen in all of the macropod species described here. The monsoonal open woodland environment in which the animals were housed is not typical for northern wallaroos or black wallaroos. They tend to be solitary and secretive in nature and are also known to be anxious in captivity and wary of exposure to the public. There have been no reports of CL in wild populations of northern wallaroos, black wallaroos or agile wallabies. CL seen in these captive macropods some years after the first report of CL in Australia suggests that Leishmania is endemic to the tropical region of the NT. Moreover CL previously seen in red kangaroos was seen in two separate locations $>20 \mathrm{~km}$ apart also indicating transmission across the Darwin rural area [2]. 
The phlebotomine sand-fly vector and the natural reservoir host are yet to be identified. Phlebotomine sand-flies from the Old World genera Phlebotomus and Sergentomyia are present in Australia, but little is known about their capacity as vectors of Leishmania spp. We hypothesize that one or more native species of macropod common to the Darwin rural area are natural reservoir hosts for Australian Leishmania. Sera and ear tissue collected from agile wallabies and antilopine wallaroos (M. antilopinis) in the region are currently being surveyed by ELISA and real-time PCR for evidence of Leishmania infection. Further research is vital in determining the reservoir status of these animals and other native marsupials found in the tropical north of Australia.

To date, no human cases of CL caused by the Australian species of Leishmania have been reported. Phlebotomine sand-flies of Australia are not known to bite humans so transmission is an unlikely event, although the vector of Leishmania in Australia is still unknown [5]. However, leishmaniasis in travellers and migrants who have travelled through or resided in endemic countries does occur in Australia. The Women's and Children's Hospital in Adelaide, South Australia, reported CL in Afghani refugees who had been in Australia up to 5 months before receiving treatment [6]. Royal Darwin Hospital in the NT treated a patient for CL caused by L. braziliensis who had been travelling for 1 year through South America [7]. In addition, the Darwin region has recently seen an increase in the number of refugees arriving from Sudan, a country that is endemic for VL. Darwin is also home to a number of Australian Defence Force personnel and CL has been observed in some soldiers returning from war zones such as Iraq and Afghanistan. Furthermore, in November 1999, 46 cases of CL were reported from in East Timor [8], which is only $640 \mathrm{~km}$ northwest of Darwin. The causative Leishmania sp. was undetermined.

VL in dogs could be another source for the introduction of an exotic Leishmania sp. into Australia. Currently all dogs are required to have a negative result for leishmaniasis prior to importation into Australia. The dogs are screened within 30 days prior to export by an indirect fluorescent antibody test (IFAT), or ELISA, as described in the Office International des Epizooties Manual [9]. Although the likelihood for an exotic introduction through imported dogs is low, consideration should be made for false-negative results which can occur using these tests. Leontides et al. surveyed 73 dogs from Greece for Leishmania spp. infection and found $63 \%$ positive by a bone marrow PCR, with only $12.3 \%$ being positive by IFAT. Dogs can carry the Leishmania parasite for months before there are signs of seroconversion [10].

This report of CL in northern wallaroos, a black wallaroo and agile wallabies highlights the importance of continued research into the life-cycle of Leishmania in Australia. It also demonstrates that 'as yet undetermined' Leishmania-transmitting vectors are endemic in Australia. The introduction of an exotic parasite into Australia, although seemingly unlikely, could have significant effects on native wildlife, livestock and public health.

\section{ACKNOWLEDGEMENTS}

This work was supported by the Australian Biosecurity Co-operative Research Centre for Emerging Infectious Disease. We thank Dr Stephen Cutter for recognizing and providing samples for the initial diagnosis of the disease in northern wallaroos.

\section{DECLARATION OF INTEREST}

None.

\section{REFERENCES}

1. Desjeux P. Leishmaniasis: current situation and new perspectives. Comparative Immunology Microbiology and Infectious Diseases 2004; 27 : 305-318.

2. Rose K, et al. Cutaneous leishmaniasis in red kangaroos: isolation and characterisation of the causative organisms. International Journal for Parasitology 2004; 34: 655-664.

3. Dawson TJ. Kangaroos: Biology of the Largest Marsupials. New York: Cornell University Press, 1995, pp. 19-25.

4. Marfurt J, et al. Identification and differentiation of Leishmania species in clinical samples by PCR amplification of the miniexon sequence and subsequent restriction fragment length polymorphism analysis. Journal of Clinical Microbiology 2003; 41: 3147-3153.

5. Lewis DJ, Dyce AL. Taxonomy of the Australasian Phlebotominae (Diptera: Psychodidae) with revision of genus Sergentomyia from the region. Invertebrate Taxonomy 1988; 2: 755-804.

6. Storer E, Wayte J. Cutaneous leishmaniasis in Afghani refugees. Australian Journal of Dermatology 2005; 46: 80-83.

7. Maguire GP, et al. New World cutaneous leishmaniasis imported into Australia. Pathology 1998; 30: 73-76. 
8. Chevalier B, et al. Report of the first cases of cutaneous leishmaniasis in East Timor. Clinical Infectious Diseases $2000 ; 30: 840$.

9. OIE World Organisation for Animal Health. Leishmaniosis, chapter 2.2.11. In: Manual of Diagnostic Tests and Vaccines for Terrestrial Animals, 5th edn, 2004
(http://www.oie.int/fr/normes/mmanual/A_00050.htm). Accessed September 2008.

10. Leontides LS, et al. A cross-sectional study of Leishmania spp. infection in clinically healthy dogs with polymerase chain reaction and serology in Greece. Veterinary Parasitology 2002, 109: 19-27. 